\title{
A Free-Access Segmented Coplanar Waveguide for On-Body Communication
}

\author{
Tran Thi Lan, Member, IEEE, Yuka Shinozaki, Member, IEEE, \\ Takuya Okura, Member, IEEE, and Hiroyuki Arai, Fellow, IEEE
}

\begin{abstract}
A segmented coplanar waveguide (SCPW) is presented in this paper to improve the propagation characteristics of on-body communication. The SCPW is connected electromagnetically by two half-wavelength resonators arranged on both sides of the center line. The resonators provide the function to couple the SCPW with the top and bottom antennas at arbitrary positions along the line, which is a unique feature of the proposed geometry. The basic performance of the SCPW was confirmed at $5.12 \mathrm{GHz}$ by a thin dielectric substrate and at 2.45 GHz by a flexible paper substrate with a silver-ink conductor. The propagation loss is reduced more than $20 \mathrm{~dB}$ by this SCPW in on-body communication between abdomen and back sides, which is confirmed by the measurement using a phantom.
\end{abstract}

Index Terms-On-body communication, coplanar waveguide, electromagnetic coupling, smart suit, transmission line.

\section{INTRODUCTION}

$\mathrm{W}$ IRELESS body area networks (WBANs) have a lot of applications from healthcare and telemedicine up to fitness and sports training, interactive gaming, and personal information sharing and authentication. WBANs can also be deployed in life-threatening environments to help protect soldiers, first responders and deep-sea or space explorers. Therefore, technologies to improve the reliability of WBANs are always attracted by researchers [1].

Some communication standards are deployed for WBANs such as Bluetooth, Zigbee, and UWB (Ultra Wide Band). One of the most important issues is the reliability of the communication link between two nodes in a non-line-of-sight (NLOS) environment due to the heavy attenuation by a human body. An easy solution for this problem is by utilizing flexible cables connecting two nodes, but, the system will become complicated by the presence of many connectors and cables, thus making users of the sensors uncomfortable, especially for moving users. Wireless connections are appropriate for on-body sensors, and the propagation loss reduction between sensors is strongly requested for low power operation.

The studies of propagation loss of the links on body suggest that the loss depends on the position of antennas on body and body posture [2]-[4]. For example, the belt-to-chest link provides low loss while higher losses are in the links of belt-to-head and belt-to-wrist. Moreover, loss of NLOS paths is at least $45 \mathrm{~dB}$ [28]. To diminish these body effects, the ground plane size is extended to decouple the antennas with the body [5], [6], resulting in less flexibility in applications on body. Another solution is the diversity reception [2], [7]-[9] to mitigate fading effects of the propagation channels using more than two antennas at the receiver, which is beneficial for dynamic environments like moving bodies.

A new method is the use of supplementary waveguides [10], providing a coverage area around themselves and electromagnetically coupled with wireless devices at any arbitrary position. These waveguides improve the propagation characteristics of on-body antennas as compared to those in free space. The users have their own waveguides independently and a high security level can be obtained. Thus, the idea of employing of supplementary waveguides for bettering the transmission on body is a new, much simpler, and high-security solution as compared to other solutions like wired connections, diversity reception or increase of ground plane size to lower body effects, which make an on-body system becomes more complicated, less flexible and sensitive to body postures.

The smart suit in [10], a wearable sheet-shaped waveguide consisting of double-layered patch resonators creates an autonomous channel for wireless devices to reduce propagation loss caused by a human body. A drawback of the smart suit is the employment of a metallic ground plane that is less comfortable and less breathable when it is inserted into a wearable fabric. Its double-layered structure makes the mass production process difficult and expensive. To simplify this sheet waveguide, a periodic bandpass-filter microstrip transmission line is also proposed for on-body links [11], [12], [26]. However, its flexibility is still limited because of the substrate ground plane for the transmission line.

This paper proposes a novel single-layer transmission line or segmented coplanar waveguide (SCPW) with non-contact coupling for antennas to improve NLOS links of on-body wireless communication. This transmission line does not only have the above-mentioned benefits of supplementary waveguides, but also has high flexibility by applying a paper substrate with a silver-ink conductor. The geometry of the transmission line was introduced in [13]. A segmented coplanar structure is employed in the transmission line, removing a ground plane layer at the bottom. It can be easily integrated into clothes at any position and can be bent to surround a waist or a shoulder of any bodies with little influence by bending. Moreover, compared to the transmission line using a microstrip line in [11], [12], [26], the proposed transmission line has a 5 -times bigger bandwidth and the 10-times thinner substrate thickness.

The communication links discussed in this paper are (a) between sensors and (b) between sensors and antennas. The sensors are attached on the body to monitor vital data or on the 
surface of clothes to obtain outside environment data. All the sensors are assumed to include antennas to transmit or receive data. The antennas for on/off-body communications are installed into clothes or outside surface of clothes, because it is very difficult to connect sensors on the body with antenna feeding cables. A non-contact coupling between transmission lines and antennas is powerfully desired for on-body communication systems. To clarify on-body propagation scenarios, this paper investigates three cases as shown in Fig. 1, where the antennas and transmission lines are embedded inside clothes, the sensors are attached on the body or the surface of clothes, and the radiation direction of antennas is toward the transmission line. As a typical application using the proposed system, vital sensors are putted on the front side of body and antennas are on the back as shown in Fig. 2. Non-contact communication links are given by the proposed transmission line. This is to allow sensors to be deployed easily and flexibly in practice. Antennas can communicate to each other, even at different sides of the transmission line, which cannot be implemented by a microstrip line [11], [12]. Particularly, more than two sensors can be excited at the same time on the transmission line. This is more active compared to wired connections when the number of sensors is increased. The transmission line greatly enhances the transmission characteristics between the sensors on body up to more than 20 $\mathrm{dB}$ for NLOS scenario. The terminology "non-contact" implies that the antennas on body communicate with each other through the electromagnetic wave in the transmission line without being connected to the transmission line while the term "free-access" means that wireless devices can couple with the segmented coplanar waveguide at any position above it.

Section II discusses the design and fabrication of the transmission line in detail. The basic performance of the transmission line on a perfect conductor plane (PEC) is presented in Section III at two frequencies of 5.12 and 2.45 $\mathrm{GHz}$, and then this performance is investigated on a simplified liquid phantom and a body model. Section IV considers the coupling power between the transmission line and an external antenna at $2.45 \mathrm{GHz}$. In Section V, the improvement of the transmission characteristics by the transmission line on both phantom and real body is evaluated. This paper concludes in Section VI.

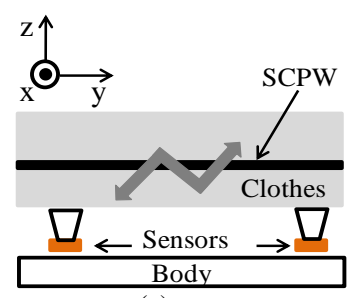

(a)

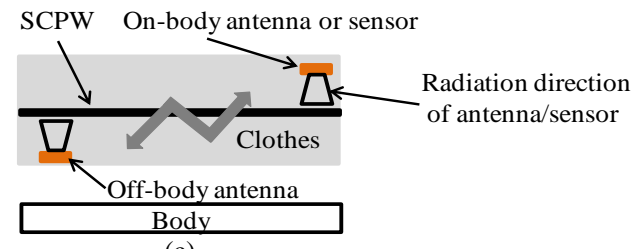

(c)

Fig. 1. Types of on-body links supported by the SCPW (side-view): (a) between sensors on body, (b) between antennas or sensors on the surface of clothes, and (c) between an off-body antenna and a sensor at the other side of the SCPW.

\section{DESIGN AND FABRICATION}

Originating from the structure of a free-access microstrip transmission line in [14], the structure is modified to a coplanar waveguide (CPW) that has performance comparable to, and sometimes even better than a microstrip line in terms of guide wavelength, dispersion, and losses [15], [27]. A special benefit of CPW is that all conductors are on one plane, including a center strip conductor with two ground planes on both sides. This makes CPW with great flexibility and the fabrication becomes much easier.

The free-access transmission line consists of some units in series. Each unit is a combination of modified two-side coupled half-wavelength $(\lambda / 2)$ resonators. Its dimension parameters are optimized so that the bandwidth is the biggest and the coupling between port 1 and port 2 is the highest. The length $l_{2}$ determines the resonance frequency of the transmission line while the gap $s_{3}$ mainly controls the input impedance of port 1 to match with $50 \Omega$ input. However, the input impedance cannot achieve to $50 \Omega$ when the width of $s_{3}$ is $0.5 \mathrm{~mm}$, which is the smallest width under the fabrication condition. Thus, gaps $g$ at two ends are introduced for easier impedance adjustment. Moreover, due to these gaps, the potentials of the two ground planes are maintained to prevent unwanted modes from propagating. The gap $s_{1}$ is optimized so that the coupling between resonators is the biggest. Other parameters are optimized to make the transmission line small such that it is suitable for on-body applications.

To extend the length of the transmission line, $n$ basic units are connected in series as shown in Fig. 3. Optimized dimension parameters of the basic unit are kept unchanged when the number of units increases. This makes the mass production process simple and not costly. Besides, together with $l_{2}$, the length $l_{3}$ is introduced to adjust the resonant frequency of $n$-unit transmission line. At first, the basic characteristics of the transmission line at $5.12 \mathrm{GHz}$ are shown using the substrate of R4737 by Panasonic with the thickness of $t_{1}=0.73 \mathrm{~mm}$, relative permittivity of $\varepsilon_{\mathrm{r} 1}=2.6$, tangent loss of $\tau_{1}$ $=0.007$, and $0.035-\mathrm{mm}$ copper conductive layer. For the flexible transmission line and the fabrication error reduction at $5.12 \mathrm{GHz}$, the performance of a paper substrate at $2.45 \mathrm{GHz}$ is examined, where the thickness of $t_{2}=0.18 \mathrm{~mm}, \varepsilon_{\mathrm{r} 2}=2.8, \tau_{2}=$ 0.15 , and a $9 \mu \mathrm{m}$ silver-ink conductive layer with the conductivity of $1.5 \times 10^{7} \mathrm{~S} / \mathrm{m}$. In the simulation of this study, CST Studio Suite [16] and EMPro [17] were used. The effect of dimension parameters on S-parameters of SCPW at 2.45 and $5.12 \mathrm{GHz}$ has a similar trend, and therefore, the simulation results are presented at one band to minimize the number of figures. Figs. 4 (a)-(e) show the S-parameters of SCPW for the parameters of $l_{2}, s_{3}, g, s_{1}$, and $l_{3}$, respectively. When these

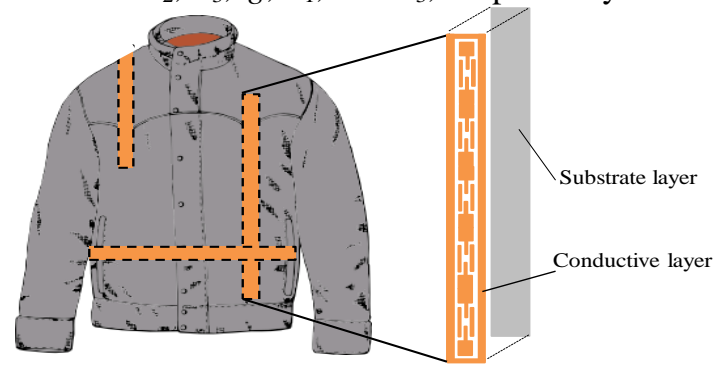

Fig. 2. The picture of a new smart suit with a single-layer non-contact coupling transmission line by using a coplanar waveguide. 
parameters are changed, the others are fixed as shown in Table I. A small change in $\mathrm{S}_{11}$ is found in the one-unit for $s_{3}$, whereas the gap $g$ changes the input impedance of port 1 of the transmission line as shown in Fig. 4 (c).

TABLE I

FABRICATION PARAMETERS OF SCPW

\begin{tabular}{|c|c|c|c|c|c|c|}
\hline \multicolumn{2}{|c|}{ Parameters[mm] } & $\boldsymbol{l}_{\mathbf{1}}$ & $\boldsymbol{l}_{\mathbf{2}}$ & $\boldsymbol{l}_{\mathbf{3}}$ & $\boldsymbol{w}_{\mathbf{1}}$ & $\boldsymbol{w}_{\mathbf{2}}$ \\
\hline \multirow{2}{*}{$\begin{array}{c}\text { Frequency } \\
{[\mathbf{G H z}]}\end{array}$} & $\mathbf{5 . 1 2}$ & 9.3 & 18.5 & 15.2 & 16 & 5 \\
\cline { 2 - 7 } & $\mathbf{2 . 4 5}$ & 18.2 & 45.6 & 36.4 & 19 & 4 \\
\hline \multicolumn{2}{|c|}{ Parameters[mm] } & $\boldsymbol{w}_{\mathbf{3}}$ & $\boldsymbol{s}_{\mathbf{1}}$ & $\boldsymbol{s}_{\mathbf{2}}$ & $\boldsymbol{s}_{\mathbf{3}}$ & $\boldsymbol{g}$ \\
\hline \multirow{2}{*}{$\begin{array}{c}\text { Frequency } \\
{[\mathbf{G H z}]}\end{array}$} & $\mathbf{5 . 1 2}$ & 1 & 1 & 1 & 0.5 & 0.5 \\
\cline { 2 - 8 } & $\mathbf{2 . 4 5}$ & 2 & 1.6 & 1 & 0.5 & 0.5 \\
\hline
\end{tabular}

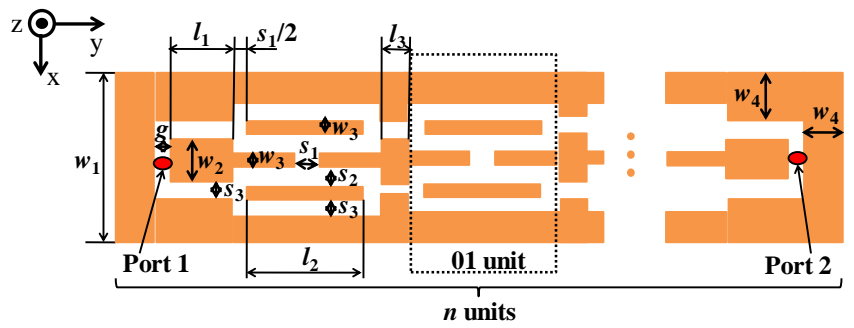

(a) Segmented CPW

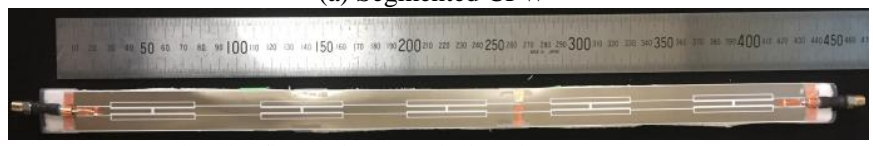

(b) The five-unit transmission line at $2.45 \mathrm{GHz}$

Fig. 3. Geometry of transmission line.

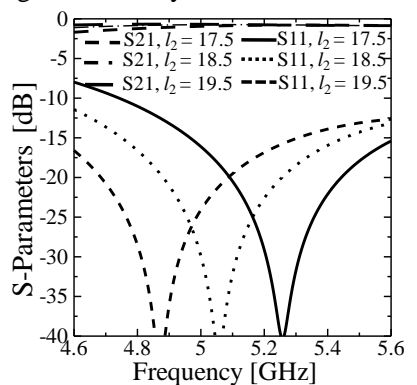

(a) Effect of $l_{2}$

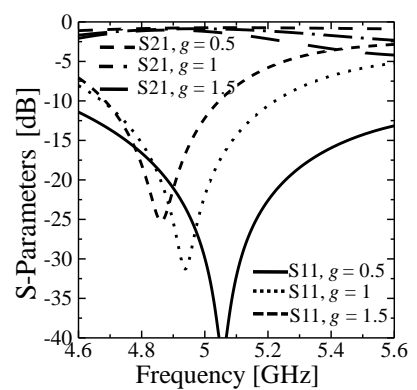

(c) Effect of $g$

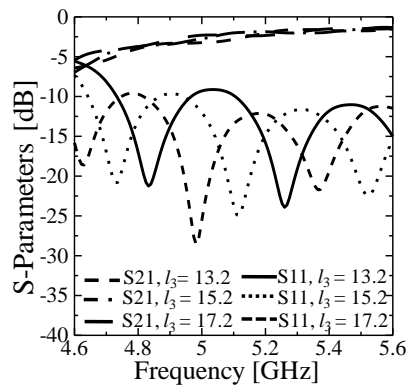

(e) Effect of $l_{3}$ at $5.12 \mathrm{GHz}$

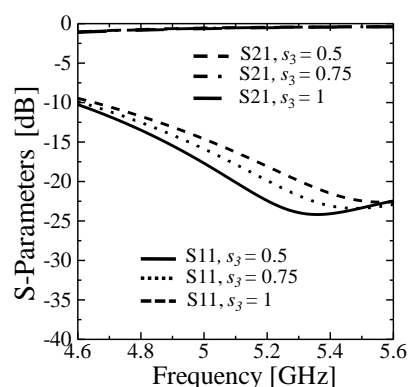

(b) Effect of $s_{3}$ (no gap $g$ )

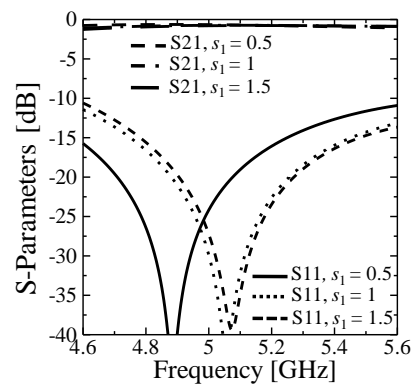

(d) Effect of $s_{1}$

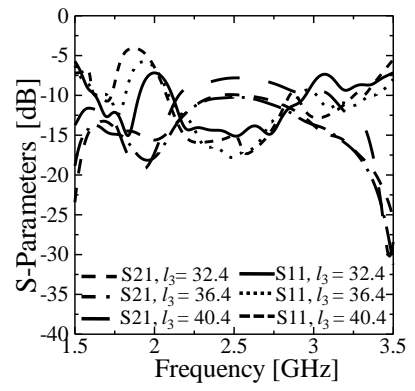

(f) Effect of $l_{3}$ at $2.45 \mathrm{GHz}$
Fig. 4. Effect of $l_{2}, s_{3}, g$ and $s_{1}$ on S-parameter of the one-unit SCPW at 5.12 $\mathrm{GHz}$ and the effect of $l_{3}$ on the five-unit SCPW.
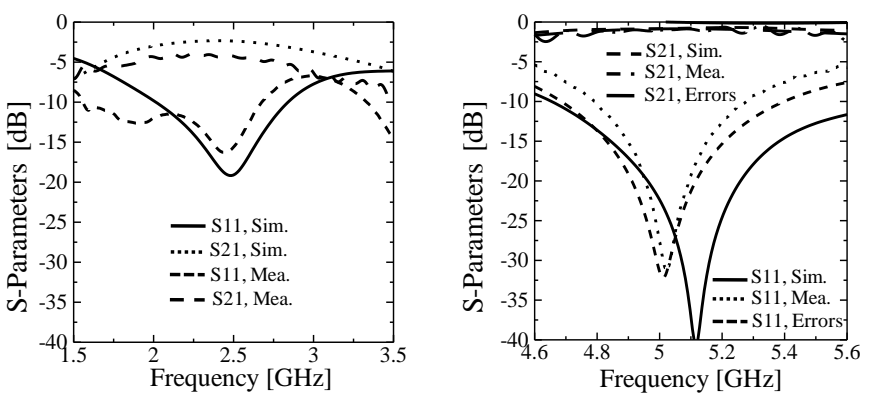

(a) $n=1$
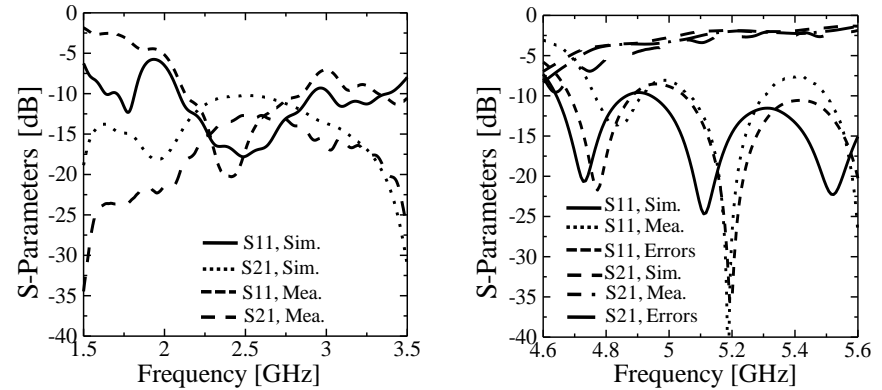

(b) $n=5$

Fig. 5. S-parameter characteristics of $n$-unit transmission line at 2.45 and 5.12 $\mathrm{GHz}$.

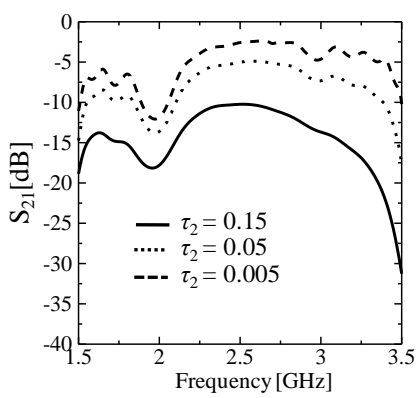

Fig. 6. Effect of the tangent loss on $\mathrm{S}_{21}$ at $2.45 \mathrm{GHz}$.

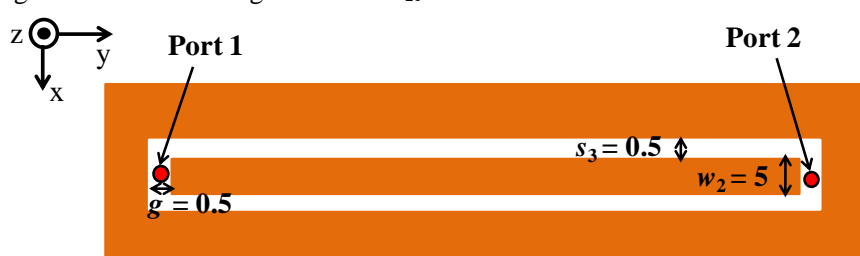

Fig. 7. Simple coplanar waveguide.

TABLE II

A COMPARISON BETWEEN THE FIVE-UNIT SCPW AND A SIMPLE CPW

\begin{tabular}{|c|c|c|c|}
\hline $\begin{array}{c}\text { Frequency } \\
\text { [GHz] }\end{array}$ & Types of CPW & S21 [dB] & $\begin{array}{c}\text { Radiation loss } \\
{[\%]}\end{array}$ \\
\hline 2.45 & SCPW & -10.3 & 21.07 \\
\cline { 2 - 4 } & Simple CPW & -6.95 & 0.186 \\
\hline \multirow{2}{*}{5.12} & SCPW & -1.97 & 20.18 \\
\cline { 2 - 4 } & Simple CPW & -0.78 & 2.18 \\
\hline
\end{tabular}

\section{BASIC PERFORMANCE OF SCPW}

The S-parameter characteristics of the transmission lines with one and five units at 2.45 and $5.12 \mathrm{GHz}$ are revealed in Fig. 5. When the number of units increases, the bandwidth of $S_{11}$ increases slightly, and the transmitted power $S_{21}$ decreases mostly by the dielectric loss of substrate and radiation loss. This is proven in Fig. 6 and Table II, and a comparison between the five-unit SCPW and a simple CPW that has a dimension equivalent to that of the SCPW as shown in Fig. 7 is presented. Although the radiation loss of the SCPW is larger than that of 
the simple $\mathrm{CPW}$, this radiation is expected for high coupling between the SCPW and the external antennas. Besides, the SCPW has a thin substrate, a symmetric structure, and similar potentials at two ground planes by two open ends, which make the SCPW significantly reduce radiation loss of unwanted modes [27].

The measurements show a good agreement with simulation. However, a small difference between simulation and measurement can be seen. This is because the connections between the connectors and the SCPW is poor due to the use of a copper tape at $2.45 \mathrm{GHz}$. At $5.12 \mathrm{GHz}$, fabrication and material errors are the main reasons for a small frequency shift, which is confirmed by the simulation results with assumed errors given in Table III. The simulation results with the assumed errors in Fig. 5 agree very well with the measured results.

A free-access microstrip transmission line is not seriously affected by the body, because it is backed by the ground plane [14]. The proposed SCPW geometry with the ground plane placed by both sides of the center line does not have shielding effect to the body. It is embedded into clothes, making a small spacing from the body, and its minimum distance for a stable operation should be examined. To evaluate the effect of the body on the SCPW, a conducting plane with the size of $750 \times$ $400 \times 1 \mathrm{~mm}^{3}$ was used as a body under the SCPW as shown in Fig. 8 [18], [19].

TABLE III

ASSUMED FABRICATION AND MATERIAL ERRORS AT $5.12 \mathrm{GHz}$

\begin{tabular}{|c|c|c|c|c|c|c|}
\hline \multicolumn{2}{|c|}{ Parameters [mm] } & $\boldsymbol{\varepsilon}_{\mathrm{r} \mathbf{1}}$ & $\boldsymbol{g}$ & $\boldsymbol{s}_{\mathbf{3}}$ & $\boldsymbol{l}_{\mathbf{2}}$ & $\boldsymbol{l}_{\mathbf{3}}$ \\
\hline \multicolumn{2}{|c|}{ No errors } & 2.6 & 0.5 & 0.5 & 18.5 & 15.2 \\
\hline \multirow{2}{*}{ Errors } & 1 unit & 2.5 & 0.7 & 0.43 & 18.3 & \\
\cline { 2 - 7 } & 5 units & 2.5 & 0.485 & 0.7 & 18.5 & 15.6 \\
\hline
\end{tabular}

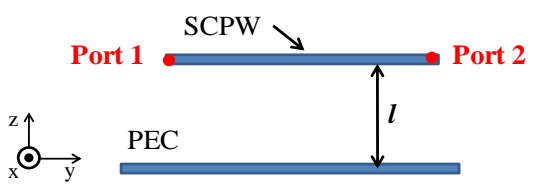

Fig. 8. Model used to consider the basic performance of the transmission line.

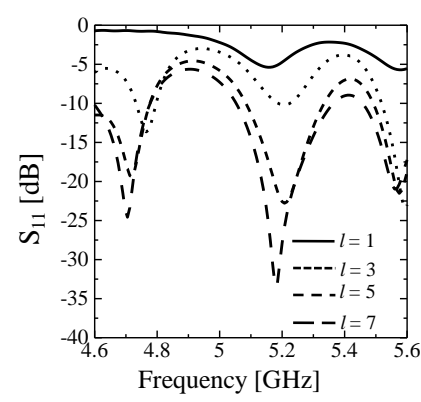

(a) $\mathrm{S}_{11}$ for $l$

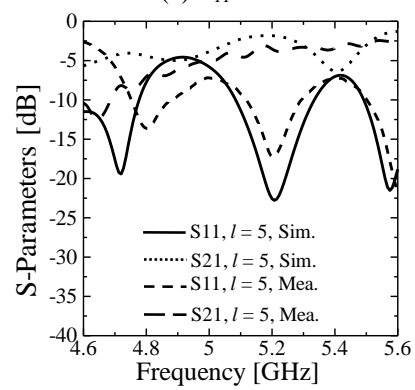

(c) Measured $\mathrm{S}_{11}$ and $\mathrm{S}_{21}$

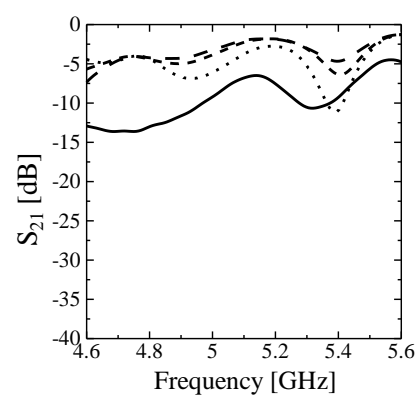

(b) $\mathrm{S}_{21}$ for $l$

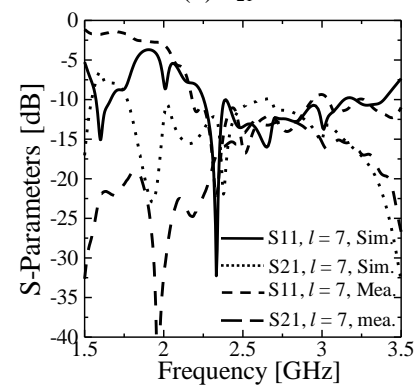

(d) Measured $\mathrm{S}_{11}$ and $\mathrm{S}_{21}$
Fig. 9. Basic performance of the transmission line.

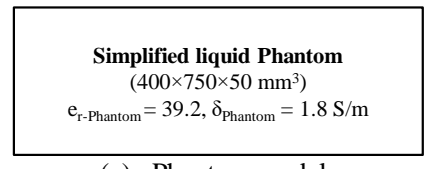

(a) Phantom model

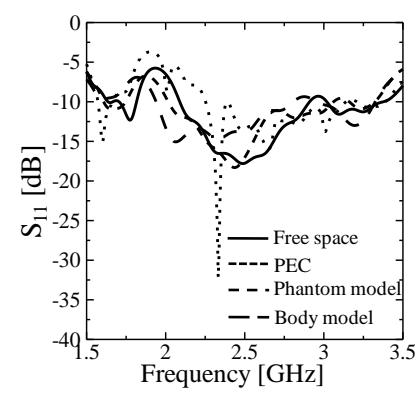

(c) $\mathrm{S}_{11}$ (b) Body model

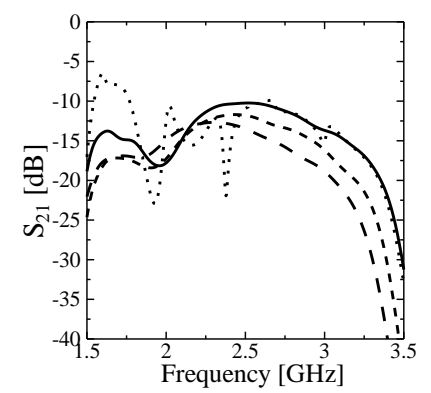

(d) $\mathrm{S}_{21}$

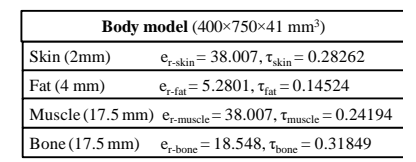

Fig. 10. S-parameters of the SCPW on a simplified liquid phantom and a body model with a distance of $7 \mathrm{~mm}$.

The S-parameters with the height $l$ changes from 1 to $7 \mathrm{~mm}$ of the SCPW at $5.12 \mathrm{GHz}$ are shown in Fig. 9. The effect of the conducting plane decreases for large $l$, and is neglected for $l \geq 5$ $\mathrm{mm}$ at $5.12 \mathrm{GHz}$. This is also verified by the measured results as presented in Fig. 9 (c). For the $2.45 \mathrm{GHz}$ band, the effect of ground plane is insignificant when the distance of $l$ is equal or greater than $7 \mathrm{~mm}$ as shown in Fig. 9 (d), where the results are similar to those in the free space as in Fig. 5 (b). Therefore, the basic performance of SCPW is not affected by body when it is placed above the body with a few millimeters spacing. This distance decreases when the operating frequency increases because the electrical distance between the body and the transmission line increases. The value of $l=7 \mathrm{~mm}$ was used for the evaluations in the next sections at the frequency of 2.45 $\mathrm{GHz}$.

The performance of the transmission line on the PEC plane is compared to those on a simplified liquid phantom [20] and a body model [21] instead of a real body with the same distance of $l=7 \mathrm{~mm}$. The simplified liquid phantom and the body model with electrical parameters are shown in Figs. 10 (a)-(b). For simplicity to show the fundamental results of the SCPW, constant values are chosen for biological tissues. In Figs. 10 (c)-(d), the S-parameters of the transmission line on the simplified phantom and the body model are similar to those in the free space as compared to those on the PEC plane. As a result, the PEC plane is the worst case for the evaluation of the performance of the transmission line. However, the PEC plane was used for easy measurement in the next sections.

Both the SCPW and the PEC plane are bent as shown in Fig. 11 (a), where two $90^{\circ}$ corners are made. The length of the upper part is equivalent to the length of two units and the length of the side part is equal to the length of 1.5 units. The S-parameters of the bent SCPW are shown in Figs. 11 (b)-(d). It is proved from the simulation results that the S-parameters of the bent SCPW is almost similar to those without bending, or the performance of the SCPW has a little influence by bending.

\section{THE COUPLING BETWEEN THE SCPW AND AN EXTERNAL ANTENNA}

The coupling with a square patch antenna [22] at any arbitrary position of the SCPW is discussed in this section at the 
frequency of $2.45 \mathrm{GHz}$.

The square patch antenna is widely-used as printed antennas for on-body communication and fed by a microstrip line with the ground plane size of $50 \mathrm{~mm} \times 50 \mathrm{~mm}$, and its substrate is similar to the SCPW at $5.12 \mathrm{GHz}$. The geometry and S-parameters of the patch antenna are illustrated in Fig. 12. The SCPW height from the PEC plane is $l=7 \mathrm{~mm}$ and its effect can be neglected as discussed in the previous section. The antennas are placed below the transmission line to emulate the coupling between the SCPW and the sensors on the body. One end of the SCPW was used as an input port 1 and the other port 2 was terminated to a $50 \Omega$ dummy load. The patch antenna feed is denoted as port 3 as illustrated in Fig. 13.

By changing the distance of $h$ between the antenna and the transmission line, and moving the antenna along the transmission line in $30 \mathrm{~mm}$ intervals, the coupling $\mathrm{S}_{31}$ between port 1 and 3 was simulated and measured. The simulation and measurement results for $h=1$ and $3 \mathrm{~mm}$ are shown in Fig. 12, where the dots were exact simulated and measured results at the positions of $y_{1}$ and the lines are approximated curves to estimate the coupling factor easily. An exponential function is used for the curves as below:

$$
\mathrm{S}_{31}=\mathrm{A} \times y_{1}^{\mathrm{b}}
$$

The values of $\mathrm{A}$ and $\mathrm{b}$ are constants to approximate the data by the function LINEST in Microsoft Excel 2007 [23], where the least squares method was used [24]. Data fluctuation in Fig. 14 is assumed to be caused by the strong coupling between SCPW and the antenna, and its variation range is reduced for large $h$. The measurement errors of several data are caused by inaccurate $y_{1}$ due to the effect of the cable and the connector, while the approximation curves in Fig. 14 are almost similar in both simulation and measurement.

This coupling power between port 1 and port 3 is higher than those of on-body propagation in [2]-[4], considering the fluctuation and the loss of paper substrate, which is the advantage of utilizing of the proposed SCPW.

\section{TRANSMISSION IMPROVEMENT BY SCPW}

\section{A. Transmission Improvement between the Two Patch Antennas on the PEC Plane}

This section presents the coupling between two patch antennas with or without the SCPW at $2.45 \mathrm{GHz}$. Fig. 15 shows the model used in simulation and measurement. In this model, the two patch antennas (used in Section IV) corresponding to port 3 and 4 were placed between the transmission line and the ground plane. The center of port 3 was fixed at a distance of $y_{0}$ $=30 \mathrm{~mm}$ from port 1 and port 4 was moved along the $y$-axis in $30 \mathrm{~mm}$ intervals by keeping the spacing of $h=1$ and $3 \mathrm{~mm}$. The distance between the centers of two patch antennas is denoted as $y_{2}$. Port 1 and 2 of the line edges are not terminated, considering practical usage.

Fig. 16 shows the simulation and measurement results. The dots represent the exact data whilst the curves represent approximation. The transmission loss of $\mathrm{S}_{43}$ between the two patch antennas through SCPW is very small and a propagation mode is assumed to be excited on the SCPW. The coupling between two patch antennas with the SCPW is greatly improved compared to those without the SCPW. The enhancement in coupling is more than $10 \mathrm{~dB}$ for small $y_{2}$ and 20 $\mathrm{dB}$ for large $y_{2}$ as shown in Fig. 16. It has been verified that the measurement results have a good agreement with simulation.

Fig. 17 shows the simulation results of the coupling between the two patch antennas in two cases as shown in Fig. 1 (b) and Fig. 1 (c), where the spacing between the antennas and the SCPW is $h=1 \mathrm{~mm}$ and the SCPW height from ground plane is $7 \mathrm{~mm}$. Fig. 17 shows that the coupling improvement is more than $10 \mathrm{~dB}$ for small $y_{2}$ and $20 \mathrm{~dB}$ for large $y_{2}$. The transmission along the SCPW enhances the coupling between the two antennas, which is a great benefit of deploying the SCPW in on-body communication.

\section{B. Coupling between the Two Antennas on a Phantom}

To emulate the measured results on real bodies, the coupling improvement by the SCPW between the two patch antennas at $2.45 \mathrm{GHz}$ was demonstrated using an ultra-light carbon phantom [25]. The upper body phantom has the size of $280 \times$ $490 \times 220 \mathrm{~mm}^{3}$. The photos of the coupling measurement between the two patch antennas and the two standard dipoles with the phantom are shown in Fig. 18.

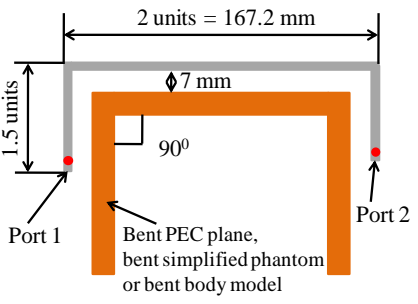

(a) Simulation model

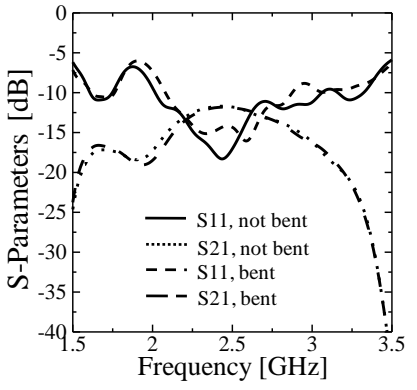

(c) SCPW above simplified phantom

Fig. 11. S-parameters of the bent SCPW.

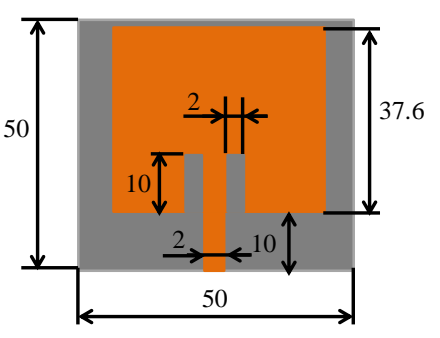

(a)

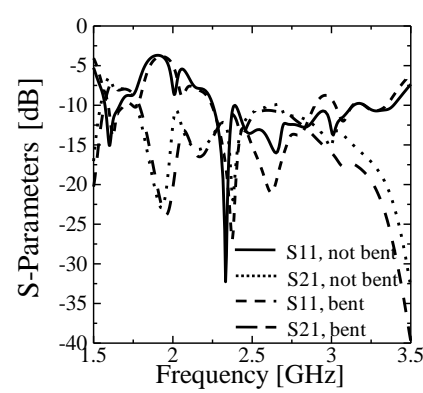

(b) SCPW above PEC

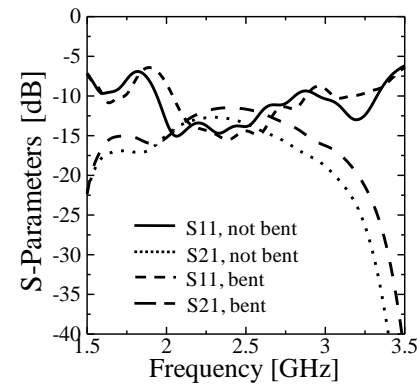

(d) SCPW above body model

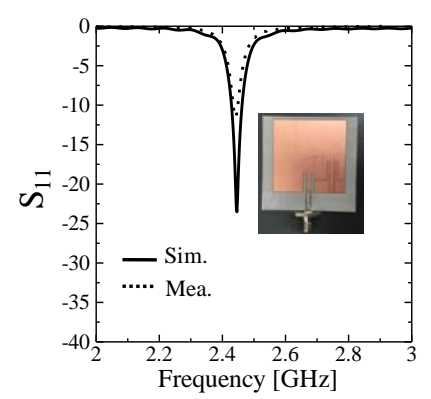

(b)
Fig. 12. Square patch antenna: (a) geometry in top view and (b) S-parameters. 


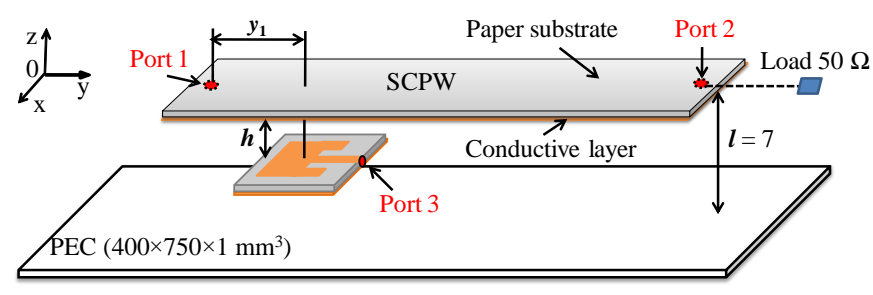

Fig. 13. Model used to consider the coupling between the patch antenna and the SCPW.

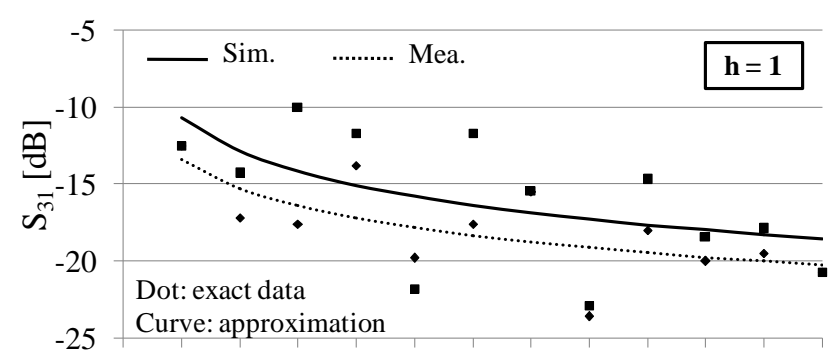

$0 \quad 30 \quad 60 \quad 90120150180210240270300330360$ Distance of $y_{1}[\mathrm{~mm}]$

(a) $h=1$

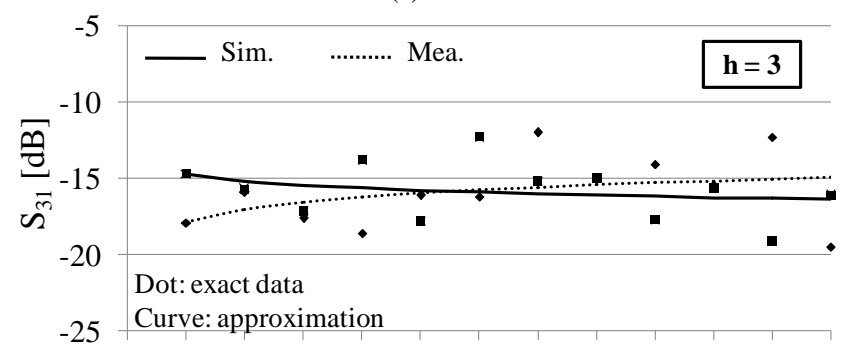

$0 \quad 30 \quad 60 \quad 90120150180210240270300330360$ Distance of $y_{1}[\mathrm{~mm}]$

(b) $h=3$

Fig. 14. Coupling power between the patch antenna and the SCPW on the PEC plane at $2.45 \mathrm{GHz}$.

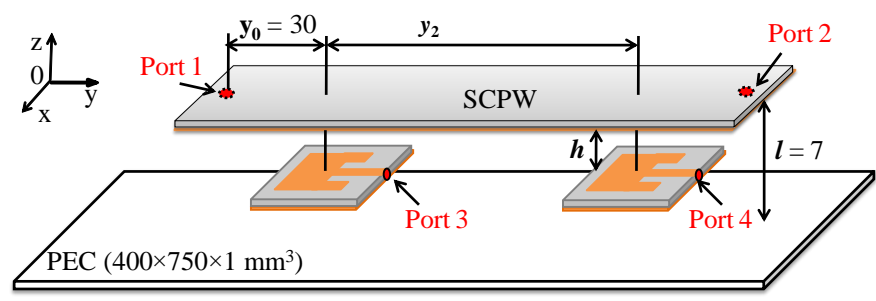

Fig. 15. Model used to evaluate the transmission loss between the two patch antennas with SCPW on the PEC plane.

Two cases were measured: In the first case, two patch antennas were placed in front of the phantom with the distance of $210 \mathrm{~mm}$ and in the second case, one antenna was putted on the front side and the other was on the back. For both cases, the SCPW was placed $7 \mathrm{~mm}$ above the phantom while the two antennas are $1 \mathrm{~mm}$ below SCPW. The dipoles are oriented in horizontal direction, being $3 \mathrm{~mm}$ below the SCPW due to the size of feeding connectors. The measured data are given in Fig. 19. The coupling characteristics using the SCPW have greatly improved, accounting for more than $20 \mathrm{~dB}$ in comparison with those without the SCPW. The improved characteristics were summarized in Table IV. Through the measured, the advantages of using the SCPW and its effectiveness for on-body communication was proved.

\section{Coupling between the Two Antennas on a Real Body}

In this section, the coupling between two patch antennas and two standard dipoles on a real body were measured to confirm the transmission improvement by the SCPW. The body is $1.7 \mathrm{~m}$ in height and $55 \mathrm{~kg}$ in weight. The measurement setting is similar to that of the phantom in the previous section. The measured results are revealed in Fig. 20. The transmission gains are also given in Table IV.

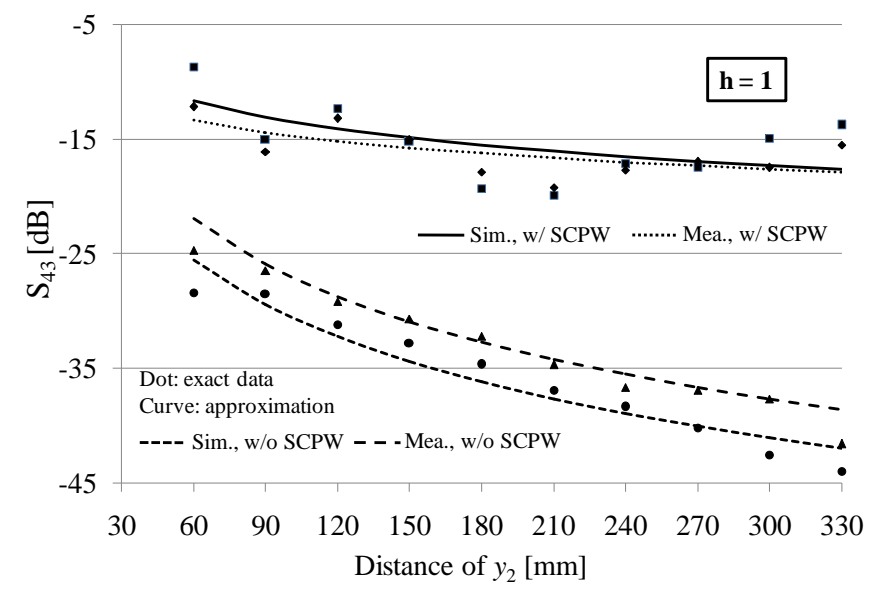

(a) $h=1$

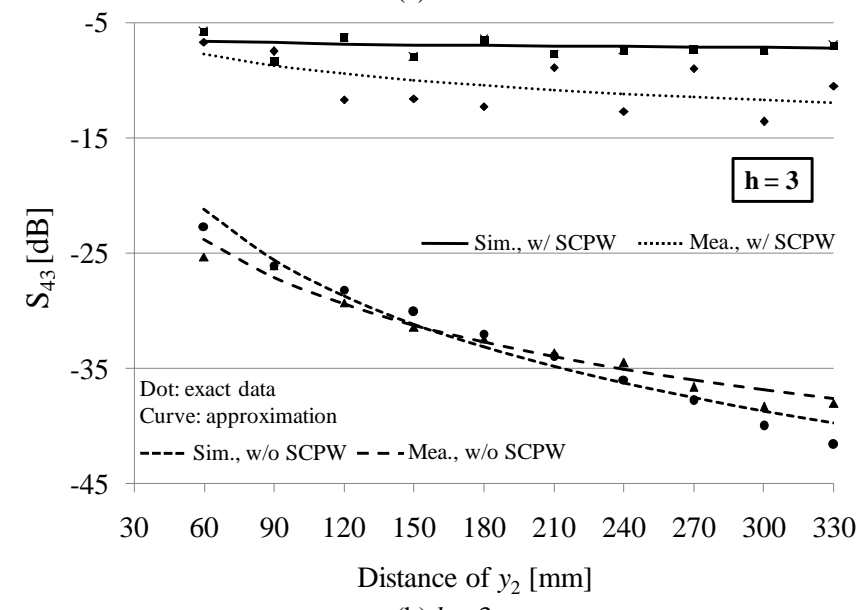

(b) $h=3$

Fig. 16. Coupling characteristics between the two patch antennas with and without the SCPW at $2.45 \mathrm{GHz}$.

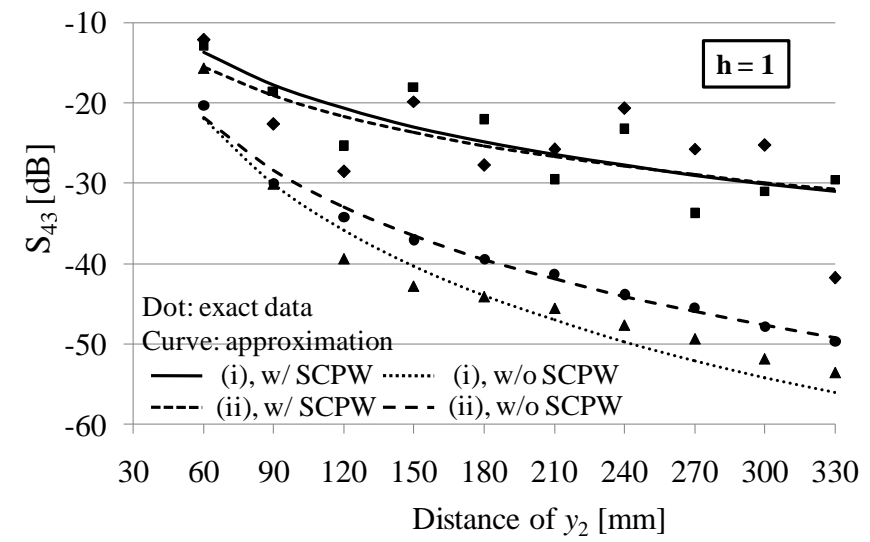

Fig. 17. Coupling between the two patch antennas with and without the SCPW at $2.45 \mathrm{GHz}$ : (i) Two antennas placed above the SCPW (Fig.1 (b)) and (ii) One antenna placed below and the other placed above the SCPW (Fig. 1 (c)). 


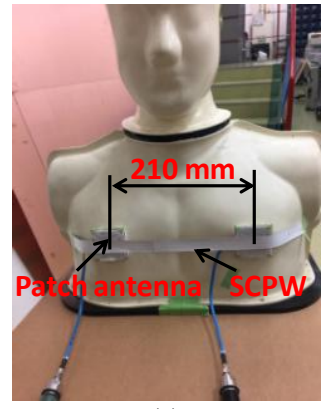

(a)

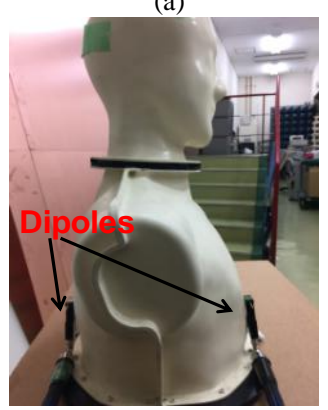

(c)

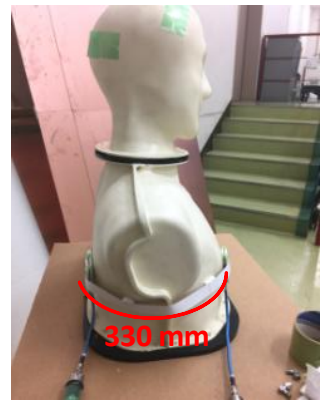

(b)

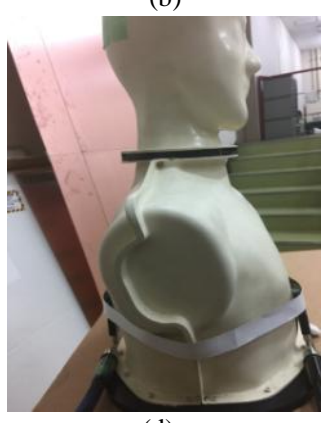

(d)
Fig. 18. Photos of the measurement using a phantom: (a) Two patch antennas putted in front of the phantom with the SCPW, (b) One antenna placed at the front and one antenna placed at the back, (c) Two standard dipoles without SCPW, and (d) two dipoles with SCPW.

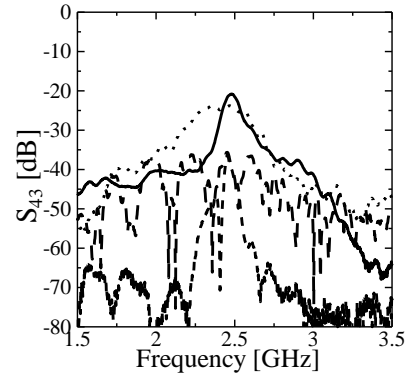

(a)

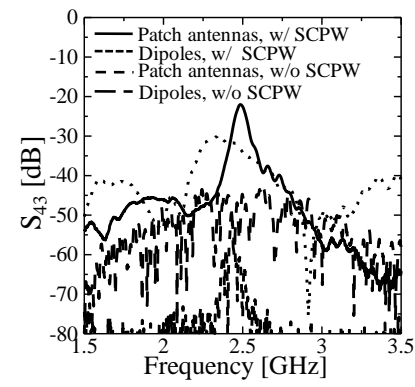

(b)
Fig. 19. Transmission losses between the two antennas on the phantom with and without the SCPW: (a) Two antennas placed in front of the phantom and (b) one antenna placed at the front and one antenna placed at the back of the phantom.

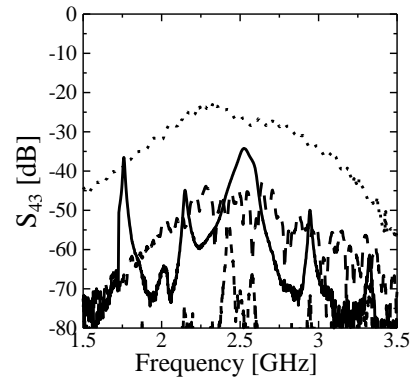

(a)

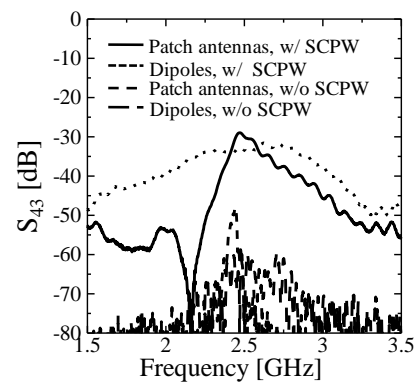

(b)
Fig. 20. Transmission losses between two antennas on the real body with and without the SCPW: (a) Two antennas placed in front of the body and (b) one antenna placed at the front, one antenna placed at the back of the body.

From the measured results on the body in Table IV, it can be observed that the coupling is improved greatly, which is similar to the measured results using the phantom. However, these improvements are similar to the two patch antennas in both cases of LOS and NLOS. This may be because of the imperfections in measurement due to the vibrations as heart beats or breathing of the body.
TABLE IV

THE TRANSMISSION GAIN BY THE SCPW AT 2.45 GHZ

\begin{tabular}{|c|c|c|c|c|c|}
\hline \multicolumn{6}{|c|}{ Two antennas are in front of the phantom/body } \\
\hline \multirow{2}{*}{\multicolumn{2}{|c|}{$\begin{array}{l}\text { Phantom/Body } \\
\text { Type of antennas }\end{array}$}} & \multicolumn{2}{|c|}{ Phantom } & \multicolumn{2}{|c|}{ Body } \\
\hline & & Patch & Dipole & Patch & Dipole \\
\hline \multirow{2}{*}{$\mathbf{S}_{\mathbf{4 3}}$} & w/ SCPW & -22 & -24.2 & -41.4 & -25.5 \\
\hline & w/o SCPW & -37.1 & -35.7 & -61.7 & -45.6 \\
\hline \multicolumn{2}{|c|}{ Transmission gain [dB] } & 15.1 & 11.3 & 20.3 & 20.1 \\
\hline \multicolumn{6}{|c|}{ One antenna is in front of the phantom/body, the other is back } \\
\hline & & \multicolumn{2}{|c|}{ Phantom } & \multicolumn{2}{|c|}{ Body } \\
\hline \multicolumn{2}{|c|}{ Type of antennas } & Patch & Dipole & Patch & Dipole \\
\hline \multirow{2}{*}{$\mathbf{S}_{\mathbf{4 3}}$} & w/ SCPW & -25.7 & -31.8 & -29.8 & -33.9 \\
\hline & w/o SCPW & -56.2 & -58.8 & -49.9 & -66.2 \\
\hline \multicolumn{2}{|c|}{ Transmission gain [dB] } & 29.5 & 27 & 20.1 & 32.3 \\
\hline
\end{tabular}

\section{CONCLUSION}

A novel single-layer non-contact coupling transmission line using a SCPW has been proposed. Its performance was verified by the measurement results on the PEC plane, a phantom and a real body. Due to many advantages such as easy fabrication by using printing technology, high flexibility, wide bandwidth, high transmission gain, and no sensitivity with bending, SCPW is really effective for on-body links when it is integrated into clothes of astronauts or rescuers. Strikingly, the transmission characteristics between the two antennas with the SCPW are significantly improved up to more than $20 \mathrm{~dB}$ for NLOS paths.

In future work, the solution to employ the SCPW in practice will be validated by covering it by thin plastic film to avoid moisture and sweat, and integrating it into clothes. Besides, specific absorption rate (SAR) also will be evaluated in detail although it is not seriously considered in this study as the SCPW is placed far from the body and the transmitting power of the sensors or antennas on body is usually very low.

\section{REFERENCES}

[1] B. Antonescu and S. Basagni, "Wireless body area networks: Challenges, trends and emerging technologies," [Online]. Avilable: http://www.ece.neu.edu/fac-ece/basagni/papers/AntonescuB13.pdf

[2] P. S. Hall, Y. I. Nechayev, C. C. Constantinou, Y. Hao, A. Alomaainy, R. Dubrovka and C. G. Parini, "Antennas and Propagation for on-body communication system," IEEE Antennas and Propagation Magazine, Vol. 49, No. 3, pp. 41-58, 2007.

[3] V. Pleskachev, I. Vendik, O. Vendik, V. Kirillov, P. Turalchuk and M. Odit, "On-body surface electromagnetic wave propagation: Modeling and Measurements", 10th EuCAP, 2016.

[4] A. Pellegrini, A. Brizzi, L. Zhang, K. Ali, Y. Hao, X. Wu, C. C. Constantinou and et al., "Antennas and Propagation for Body-Centric Wireless Communications at Millimeter-Wave Frequencies: A Review," IEEE Antennas and Propagation Magazine, Vol. 55, no. 4, pp. 262-287, Aug. 2013.

[5] M. Koohestani, J. F. Zürcher, A. A. Moreira and A. K. Skrivervik, "Anovel, low-profile, vertically-polarized UWB antenna for WBAN," IEEE Trans. Antennas Propag., Vol. 62, no. 4, pp. 1888-1894, Apr. 2014.

[6] P. B. Samal, P. J. Soh and G. A. E. Vandenbosch, "UWB All-Textile Antenna With Full Ground Plane for Off-Body WBAN Communications," IEEE Trans. Antennas Propag., vol. 62, no. 4, pp. 102-108, Jan. 2014.

[7] A. Michalopoulou, A. A. Alexandridis, K. Peppas, T. Zervos, F. Lazarakis, K. Dangakis and D. I. Kaklamani, "Statistical Analysis for On-Body Spatial Diversity Communications at $2.45 \mathrm{GHz}$," IEEE Trans. Antennas Propag., Vol. 60, no. 8, pp. 4014-4019, Aug. 2012.

[8] Q. H. Abbasi, E. Serpedin, K. Qaraqe, A. Alomainy and Y. Hao, "Multiband-OFDM based Ultra Wideband System Modelling of On/Off-Body Antenna Diversity," IEEE International Symposiumon Antennas and Propagation \& USNC/URSI National Radio Science Meeting, 2015, pp. 2363-2364. 
[9] Y. Yao, J. Zheng, and Z. Feng, "Diversity Measurements for On-Body Channels Using a Tri-Polarization Antenna at $2.45 \mathrm{GHz}, "$ IEEE antennas and wireless propagation letters, Vol. 11, pp. 1285-1288, 2012.

[10] C. Mikeka and H. Arai, "Novel wearable sensors for body area network applications," in Microwave and Millimeter Wave Circuits and Systems: Emerging Design, Technologies, and Applications, $1^{\text {st }}$ ed., John Wile\& Sons, 2012.

[11] H. Arai, "Free access transmission line for body centric communication (Invited)," 2015 IEEE MTT-S 2015 International Microwave Workshop Series on RF and Wireless Technologies for Biomedical and Healthcare Applications (IMWS-BIO), Taipei, 2015, pp. 136-137.

[12] Y. Shinozaki, T. Okura and H. Arai, "Coupling characteristics between two dipole antennas over free access transmission line using paper substrate," 2016 International Symposium on Antennas and Propagation (ISAP), Okinawa, 2016, pp. 310-311.

[13] T. L. Tran, Y. Shinozaki and H. Arai, "A Flexible Transmission Line Using Coplannar Waveguide for On-body Links," 2017 ISAP, Phuket, Thailand.

[14] T. Okura and H. Arai, "One-dimensional free access transmission line for RFID reader," IEICE comex, Vol. 2, no. 1, 7-11, 2013.

[15] Rainee N. Simons, "Conventional Coplanar waveguide," in Coplanar waveguide circuits, Components, and systems, John Wiley\&Sons, 2001.

[16] CST software. [Online]. Available: https://www.cst.com/products/cstmws

[17] EMPro software. [Online]. Available: http://www.keysight.com/en/pc1297143/empro-3d-em-simulation-software?cc=GB\&lc=eng

[18] N. P. B. Kammersgaard, S. H. Kvist, J. Thaysenand and K. B. Jakobsen, "Validity of PEC Approximation for On-Body Propagation," $10^{\text {th }} E u C A P$, Apr. 2016.

[19] Z. Ma, J. Sarrazin, L. Petrillo and T. Mavridis, "Antenna Radiation Characterization for On-Body Communication Channel Using Creeping WaveTheory," $9^{\text {th }} E u C A P$, Apr. 2015.

[20] K. Fukunaga, S. Watanabe, Y. Yamanaka, H. Asou, Y. Ishii and K. Sato, "Dielectric properties of liquid phantoms for evaluations of mobile phones," IEICE EMC'04, Sendai, Japan.

[21] M. Rizwan, M. W. A. Khan, L. Sydanheimo and L. Ukkonen, "Performance evaluation of circularly polarized patch antenna on flexible EPDM substrate near human body," 2015 LAPC.

[22] C. A. Balanis, "Microstrip antennas," in Antenna Theory, John Wiley \& Sons, $3^{\text {rd }}$ ed., pp. 816-843, 2005.

[23] FINEST function. [Online]. Available: https://support.office.com/en-us/ article/LINEST-function-84D7D0D9-6E50-4101-977A-FA7ABF772B6D

[24] J. Wolberg, "The method of least squares," in Data Analysis using the method of least squares, Springer, 2006

[25] Phantom [Online]. Avilable: http://www.mwf.co.jp

[26] Y. Shinozaki and H. Arai, "Free access transmission line using paper substrate in body area network," 2017 International Workshop on Electromagnetics: Applications and Student Innovation Competition, IEEE, 2017.

[27] K. C. Gupta, R. Garg, I. Bahl and P. Bhartia, "Microstrip lines and Slotlines," Chapter 7, $2^{\text {nd }}$ ed., Artech House, 1996.

[28] R. Chandra and A. J. Johansson, "An elliptical link loss model for wireless propagation around the human torso," $6^{\text {th }} E U C A P, 2012$.

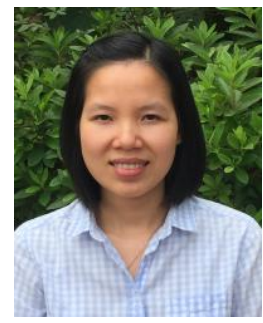

Tran Thi Lan (M'15) was born in Hai Phong, Vietnam, in 1988. She received the B.S and M.S in Telecommunication Engineering from the University of Transport and Communications in Ha Noi, Vietnam in 2011 and 2013, respectively. She works as a tutor and a lecturer at University of Transport and Communications from 2011. She got the Vietnamese government scholarship, and now is pursuing the $\mathrm{Ph}$. D. degree in Computer, Physics and Electrical Engineering at the Yokohama National University, Yokohama, Japan. Her research interests are the design of antennas for body area network (BAN), and low loss transmission lines for on-body links.

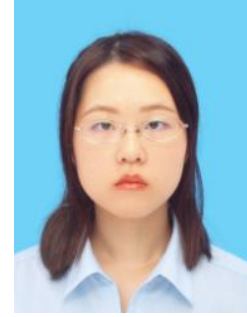

Yuka Shinozaki (M'17) received the BS degree in electrical and computer engineering from Yokohama National University in 2016. She received the ME degree from Yokohama National University in 2017. She is currently working towards the DE degree at Yokohama National University.

Her research interests include short-range wireless systems and wearable networks and microwave absorber.

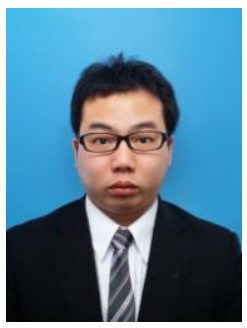

Takuya Okura (M'14) received the B.E., M.E., and D.E. degrees in electrical and computer engineering from Yokohama National University, Yokohama, Japan, in 2012, 2013, and 2016, respectively.

Since 2017, he has been with the National Institute of Information and Communications Technology (NICT), and has been engaged in research on antennas and satellite communications, including several satellite communications experiment projects. His current research interests include phased array antenna, systematic error calibration technique of array antenna, active electronically scanned array antenna for aircraft.

Dr. Okura is a member of the Institute of Electronics, Information and Communication Engineers, Japan.

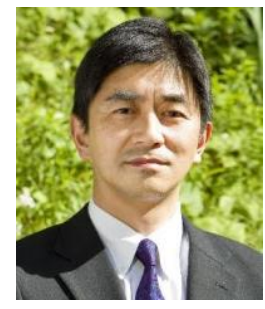

Hiroyuki Arai (F'12) received the B.E. degree in electrical and electronic engineering and the M.E. and D.E. degrees in physical electronics from the Tokyo Institute of Technology, Tokyo, Japan, in 1982, 1984, and 1987, respectively.

He was a Research Associate with the Tokyo Institute of technology. In 1989, he joined Yokohama National University, Yokohama, Japan, as a Lecturer. He was a Visiting Scholar with the University of California at Los Angeles, Los Angeles, CA, USA, in 1997. He was a Visiting Professor in 2005 and an Adjunct Professor from 2012 to 2014, respectively, with Yonsei University, Seoul, South Korea.

He is currently a Professor with the Department of Electrical and Computer Engineering, Yokohama National University. He investigated microwave passive components for high power handling applications, such as RF plasma heating in large tokamaks. He developed a flat diversity antenna for mobile telephone terminal, a polarization diversity base station antenna for Japanese PDC systems, small base station antennas of in-building microcellular system, and direction-of-arrival (DOA) estimation for cellular system. He has authored five edited books, three research book chapters, over 140 journal papers, and 1100 conference papers. His current research interests include Multiple-input and multiple-output antennas, wireless power transmission, energy harvesting in EM waves, and Electromagnetic compatibility/ Electromagnetic interference antennas.

Prof. Arai is a fellow of Institute of Electronics, Information and Communication Engineers (IEICE). He received the Young Engineers Award from the IEICE of Japan in 1989 and 
the Meritorious Award on Radio by the Association of Radio Industries and Businesses in 1997 for the development of polarization diversity antenna, in 2006 for the development of DOA estimation system, and in 2011 for the development of light weight phantom. He was the Editor-in-Chief of IEICE Transactions on Communications from 2005 to 2007, and a Chair of the IEEE AP-S Japan Chapter from 2009 to 2010. He was an Associate Editor of the IEEE TRANSACTIONS ON ANTENNAS AND PROPAGATION from 2011 to 2013 and the Chair of the Technical Group on Antennas and Propagation of IEICE from 2013 to 2014. 